\title{
Present Status and Progress towards Kala-Azar Elimination Programme in Uttar Pradesh, India
}

\author{
Ramesh Chandra', Shaukat Kamal ${ }^{2}$, SM Singh ${ }^{3}$, Ashish Kumar ${ }^{4}$, KKMittra $^{5}, \underline{\text { Nupur Roy }}^{6}$ \\ 1,2,3,4,5 Regional Office of Health \& Family Welfare, Govt. of India, Kendriya Bhawan, $9^{\text {th }}$ Floor, Aliganj, Lucknow, Uttar Pradesh, India. \\ ${ }^{6}$ National Vector Borne Disease Control Programme, MOH \& FW, Govt. of India, 22, Sham Nath Marg, Delhi, India.
}

DOI: https://doi.org/10.24321/0019.5138.202108

\section{I $\quad \mathbf{N} \quad \mathbf{F} \quad \mathbf{O}$}

\author{
Corresponding Author: \\ Shaukat Kamal, Regional Office of Health \& Family \\ Welfare, Govt. of India, Kendriya Bhawan, $9^{\text {th }}$ \\ Floor, Aliganj, Lucknow-226 024, India. \\ E-mail Id: \\ shaukatkamal25@yahoo.in \\ Orcid Id: \\ https://orcid.org/0000-0002-3358-1572 \\ How to cite this article: \\ Chandra R, Kamal S, Singh SM, Kumar A, Mittra \\ KK, Roy N. Present Status and Progress towards \\ Kala-Azar Elimination Programme in Uttar \\ Pradesh, India. J Commun Dis 2021; 53(1): 45-57. \\ Date of Submission: 2021-02-18 \\ Date of Acceptance: 2021-03-25
}

\section{$\begin{array}{llllllll}\mathbf{A} & \mathbf{B} & \mathbf{S} & \mathbf{T} & \mathbf{R} & \mathbf{A} & \mathbf{C} & \mathbf{T}\end{array}$}

Kala-azar (KA) and Lymphatic Filariasis (LF), two out of the six vector borne diseases slated for elimination from the country by Government of India. Kala-azar or Visceral Leishmaniasisis prevalent in the eastern part of Uttar Pradesh mainly in the districts bordering to Bihar, the state known for high endemicity of the disease. This disease has been targeted for elimination not only from thestate butfrom the country by 2020 by bringing down the Kala-azar prevalenceto $<1$ per 10000 population at sub district/block level. The two important interventions in Kala-azar control Programme includes Indoor residual spray (IRS) and Active Case Searches (ACS) in the endemic districts. The disease has been existed since long back in the state and the efforts made towards elimination are inadequate and deviated from the guidelines laid down by Directorate of National Vector Borne Disease Control Programme (NVBDCP).If the population of the sub district/ block is considered basic unit for calculating the prevalence of Kala-azar case $<1$ per 10000 population, then the state has achieved the same and as no activity pertaining to Kala-azar intervention measure is needed but in order to achieve the real target, it is suggested to consider the population of Health Sub-Center (HSC) for calculating the parameter of Kala-azar case $<1$ per 10000 population, for which a long way is required by undertaking measures like (a) active cases search drive for at least up to three years sweeping the whole population of the district, (b) complete treatment of the KA cases (both VL \& PKDL), (c) complete coverage of population with IRS following the time line and quality of IRS as per NVBDCP guidelines, and (d) intervention activities needs to be synchronized with neighboring states or country subjecting their areas for KA elimination. Present study revealed the current status and progress towards elimination of the disease.

Keywords: Kala-azar Outbreak, Surveillance, Intervention Measures, Leishmania donovani, Phlebotamus Argentipes, Activecase Case Search 


\section{Introduction}

Uttar Pradesh is the largest state of India comprised of 75 revenue districts and area of $243,286 \mathrm{sq} . \mathrm{km}$, reflects population density of 828 persons per sq. $\mathrm{km}$. The population of Uttar Pradesh is highest 230 million population (199.81 million as per 2011 census). It has vast length and width. About two third population resides in rural areas and mainly depend upon agricultural practices as the Indo-Gangetic plain is contributing a lot in the fertile agricultural region for the development of not only the state but India as a whole. Since this area is traversed be a number of big and small rivers, provide good opportunity $\&$ facility for irrigation of the crops, horticulture fields and industry too. The state had public health problems as every third person suffered due to malaria with high mortality \& morbidity prior to the independence. Though Malaria was the major problem, yet Kala-azar and plague also posed a threat. The launch of National Malaria Control Programme (NMCP) in 1953 with organized control efforts by performing DDT 50\% wdp Indoor Insecticidal Spray (IRS) inside the human dwellings, resulted drastic decline in malaria cases, as only 0.10 million cases of malaria in India and 3365 cases left in Uttar Pradesh in 1965 , thereby eradicating the dreadful disease from almost major part of the country. The Indian sub-continent, which experienced several epidemics of Kala-azar in eastern part 1 of India at an interval of ten years and lasted in 10 years duration, ceased to occur automatically. The epidemics of plague, a zoonotic disease, were also prevented, as no death due to plague was reported after $1967^{2}$ though the activity of the bacillus responsible for plague continued in rodent host population till 1980, as the isolation of the bacillus was reported from rodent of Himachal Pradesh, thereafter no isolation of bacillus reported, only serological positivity was reported in Tatarica indica. The possible cause of check on epidemics of Kala-azar and prevention of plague epidemics may be attributed to the pressure exerted on the vectors of the two diseases by insecticidal use against malaria in public health and in agriculture, without any additional effort/ input against Kala-azar and plague vectors. The limitation of conducting IRS operations, was experienced on technical ground due to reduction in malaria cases. The handing over of malaria free areas to the basic health services in the states for maintaining the malaria free status in their area and restricted use of insecticides in public health, resulted in resurgence of the many of the vector borne diseases, among which included kala-azar too. The local and focal outbreaks of malaria with high morbidity \& mortality, occurred in different states, thereby increased the toll of malaria cases to 6.47 million in 1977 in the country. The reporting of kala-azar started by seventies and as such Bihar \& West Bengal became worst affected among all. ${ }^{1}$ The introduction of Japanese Encephalitis (JE) in north-eastern Uttar Pradesh, during
1978, swept over the affected population with IRS again, prevented the population from kala-azar but the IRS was withdrawn in midth of nineties, due to the exophilic and exophagic behavior of the JE vector. The other districts of Uttar Pradesh, not covered under IRS, reported sporadic occurrence of kala-azar cases 1 but outbreak occurred in 1987-88 in District Bhadohi (erstwhile part of district Varanasi), a place known for carpet weaving, for which looms are installed in the houses. The labour deployed in the looms, was used to come from Bihar, a highly kala-azar affected state.

Concerned with the increasing problem of Kala-azar in the country, the Government of India (GOI) launched a centrally sponsored Kala-azar Control Programme in the endemic states in 1990-91. The Gol provided drugs, insecticides and technical support and state governments provided costs involved in implementation. The program was implemented through State/ District Malaria Control Offices and the primary health care system with the existing set up of malaria programme without any additional manpower. Prior to this, the disease kala-azar was looked through general health services in the country. The programme brought a significant decline in Kala-azar morbidity, but could not sustain the pace of decline for longer period.

The National Health Policy-2002 set the goal of Kala-azar elimination in India by the year $2010^{4}$ in order to improve the health status of vulnerable groups and at-risk population living in Kala-azar endemic areas by the elimination of Kala-azar, so that it no longer remains a public health problem. The target of elimination was revised to $2015^{7-9}$ and now to $2020^{10}$ but the target has not been achieved so far, which envisage reduction in the annual incidence of Kala-azar to $<1$ case per 10,000 population at the subdistrict (block PHCs) level in Bangladesh and India and at the district level in Nepal.

The National Vector Borne Disease Control Programme (NVBDCP)is an umbrella programme for prevention and control of vector borne diseases and is subsumed under National Health Mission (NHM).Presently all programmatic activities pertaining to vector borne diseases in the country are being implemented through NVBDCP with the objectives of reducing Kala-azar in the vulnerable, poor and unreached populations in endemic areas; reducing case-fatality rates from Kala-azar to negligible level; reducing cases of PKDL to interrupt transmission of Kala-azar; and preventing the emergence of Kala-azar and HIV/ TB co-infections in endemic areas. The present paper deals with the status and efforts \& progress of the state towards the elimination of the Kala-azar disease.

\section{Methodology}

A multipronged approach was planned and implemented 
as the national strategy for elimination of Kala-azar which included: Early diagnosis \& complete case management; Integrated Vector Management and Vector Surveillance; Supervision, monitoring, surveillance and evaluation; Strengthening capacity of human resource in health; Advocacy, communication and social mobilization for behavioral impact and inter-sectoral convergence and Programme management. In order to ensure action on different approaches of the disease elimination, the Kalaazar endemic and affected district Kushinagar, Deoria, Ballia, Ghazipur, Varanasi, Jaunpur and Sultanpur were visited during 2018 \& 2019. The activities related to different approaches were observed in the districts. The observation made so in the districts were analyzed and interpreted.

\section{Result and Discussion}

Since, there was no identified programme for prevention and control of the Kala-azar disease, the number of KalaAzar (KA) cases reported by the state form various treatment hospitals in 1977, the time of implementation of modified plan of operation for malaria and when the National Vector Borne Disease Control Programme (erstwhile National Malaria Eradication Programme) started collection of information on Kala-azar (KA) cases, has been furnished through graphic presentation in the document (Figure 1). ${ }^{1-6}$ The disease is not reported from all 75 districts of the state but the maximum number of KA reporting districts in the eastern half of the state remained only 16 during last twenty five years. It is evident from the (Table 1) that only five districts namely Ballia, Ghazipur, Varanasi, Deoria and Kushinagar have been reporting Kala- azar cases regularly. In addition to this, District Sant Ravidas Nagar (Bhadohi), (which was earlier a part of district Varanasi), also experienced KA outbreak in 1987-88, resulting considerable morbidity \& mortality. ${ }^{1}$ As per the National guidelines, the state is implementing various intervention activities, which are briefly documented in this communication.

\section{Surveillance of Kala-azar (KA) Cases}

The district Ballia, Ghazipur, Varanasi, Deoria and Kushinagarare highly KA endemic districts reporting cases on regular interval, had conducted special search drive for abouta fortnight twice in a year. The hospital based surveillance has also been included in these cases. On analyzing the month wise data of KA cases of last four years, it was revealed that the reporting of KA cases remained throughout the year with increased number in the last quarter of the year and a slight rise in post spring months (Figure 2). The surveillance data in absence of the regular surveillance staff, is not sufficient to conclude the seasonality of the disease but of the disease pattern, it can be assumed that the transmission of the disease in the state is post spring and post monsoon season and the intervention activities pertaining to the elimination of the disease are being instituted by the state, keeping in view the disease transmission period.

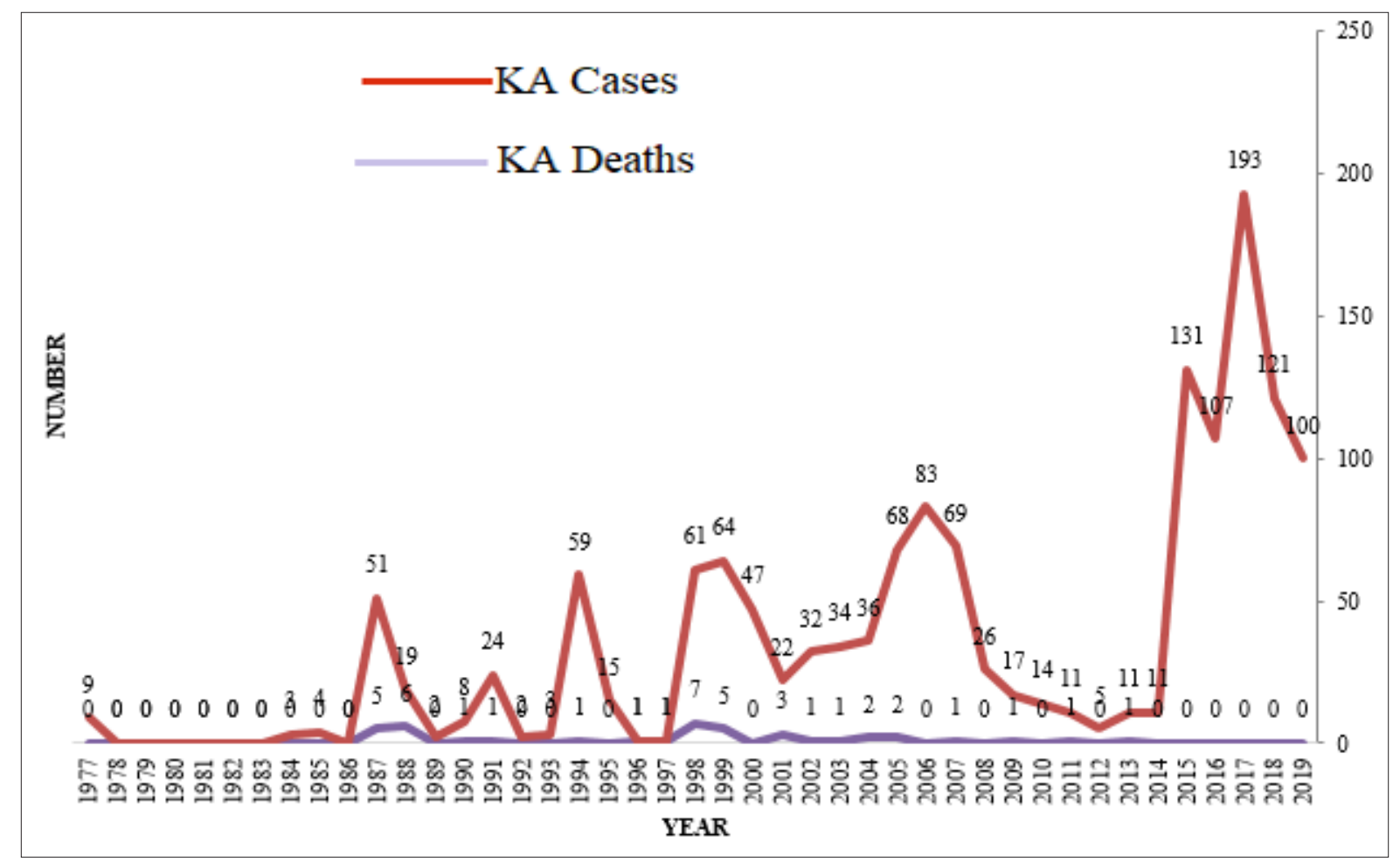

Figure I.Number of Kala-azar Cases and Deaths reported in Uttar Pradesh from 1977 to 2019 


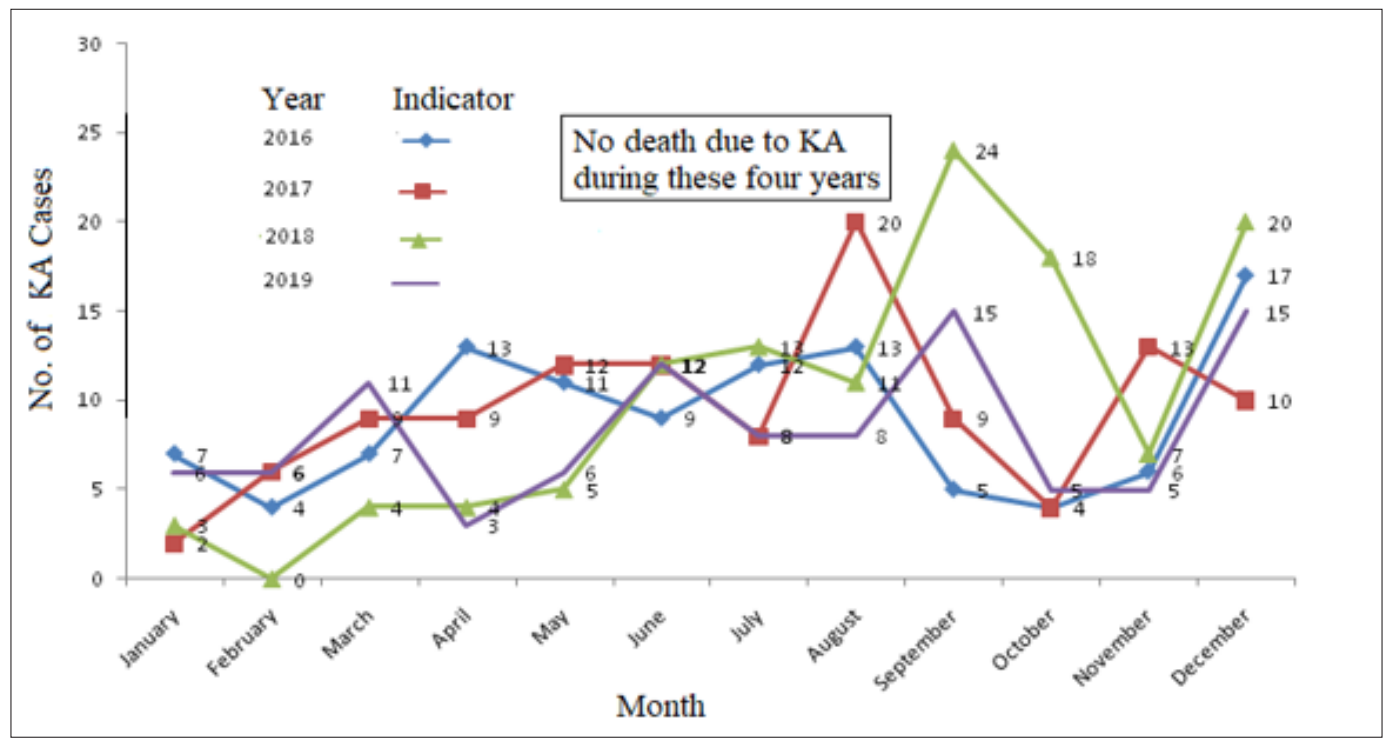

Figure 2.Seasonality of Kala-azar Cases in Uttar Pradesh from 2016-2019

Table I.District wise Status of Kala-azar Cases in Uttar Pradesh

\begin{tabular}{|c|c|c|c|c|c|c|c|c|c|c|c|c|c|c|c|c|c|c|c|c|c|c|c|c|c|}
\hline \multirow[t]{2}{*}{$\begin{array}{l}\text { S. } \\
\text { No. }\end{array}$} & \multirow[t]{2}{*}{$\begin{array}{c}\text { District/ } \\
\text { Year }\end{array}$} & \multicolumn{2}{|c|}{1994} & \multicolumn{2}{|c|}{1995} & \multicolumn{2}{|c|}{1996} & \multicolumn{2}{|c|}{1997} & \multicolumn{2}{|c|}{1998} & \multicolumn{2}{|c|}{1999} & \multicolumn{2}{|c|}{2000} & \multicolumn{2}{|c|}{2001} & \multicolumn{2}{|c|}{2002} & \multicolumn{2}{|c|}{2003} & \multicolumn{2}{|c|}{2004} & \multicolumn{2}{|c|}{2005} \\
\hline & & ث & 草 & ث & $\stackrel{*}{*}$ & ث & $\stackrel{*}{*}$ & * & 莡 & ث & 苂 & ث & 苂 & U & $\stackrel{*}{*}$ & U & $\stackrel{*}{*}$ & ث & 草 & U & 菊 & ث & $\stackrel{*}{*}$ & U & $\stackrel{*}{*}$ \\
\hline 1. & Deoria & 1 & 0 & & & & & & & & & 3 & 0 & 2 & 0 & 4 & 0 & & & & & & & & \\
\hline 2. & $\begin{array}{l}\text { Kushi- } \\
\text { nagar }\end{array}$ & & & & & & & & & 61 & 7 & 55 & 5 & 40 & 0 & 21 & 3 & 30 & 1 & 19 & 0 & 1 & 0 & 27 & 0 \\
\hline 3. & Ballia & 8 & 1 & & & 1 & 1 & & & & & 6 & 0 & 2 & 0 & & & 2 & 0 & 14 & 0 & 21 & 1 & 20 & 1 \\
\hline 4. & Mau & & & & & & & 1 & 1 & & & & & & & & & & & & & & & & \\
\hline 5. & Varanasi & 45 & 0 & 2 & 0 & & & & & & & & & & & & & & & & & 3 & 0 & 6 & 0 \\
\hline 6. & Ghazipur & 1 & 0 & & & & & & & & & & & 2 & 0 & & & & & & & & & & \\
\hline 7. & Jaunpur & 1 & 0 & & & & & & & & & & & & & & & & & & & & & & \\
\hline 8. & Chan-dauli & & & & & & & & & & & & & & & & & & & & & & & & \\
\hline 9. & Bhadohi $^{\#}$ & & & & & & & & & & & & & & & & & & & & & & & & \\
\hline 10. & Mirzapur & 1 & 0 & & & & & & & & & & & & & & & & & & & & & & \\
\hline 11. & Gora-khpur & & & & & & & & & & & & & 1 & 0 & & & & & & & & & & \\
\hline 12. & Ayodhya** & & & 13 & 0 & & & & & & & & & & & & & & & & & & & & \\
\hline 13. & Sultanpur & & & & & & & & & & & & & & & & & & & & & & & & \\
\hline 14. & Gonda & 2 & 0 & & & & & & & & & & & & & & & & & & & 11 & 1 & 15 & 1 \\
\hline 15. & Bahraich & & & & & & & & & & & & & & & & & & & & & & & & \\
\hline 16. & Rampur & & & & & & & & & & & & & & & & & & & 1 & 1 & & & & \\
\hline & Total & 59 & 1 & 15 & 0 & 1 & 1 & 1 & 1 & 61 & 7 & 64 & 5 & 47 & 0 & 25 & 3 & 32 & 1 & 34 & 1 & 36 & 2 & 68 & 2 \\
\hline & $\begin{array}{l}\text { No. of } \\
\text { Districts } \\
\text { reported } \\
\text { KA case }\end{array}$ & 7 & 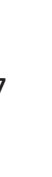 & 2 & & 1 & & 1 & & 1 & & 3 & & 5 & & 2 & & 2 & & 3 & & 2 & & 2 & \\
\hline
\end{tabular}




\begin{tabular}{|c|c|c|c|c|c|c|c|c|c|c|c|c|c|c|c|c|c|c|c|c|c|c|c|c|c|c|c|c|c|}
\hline \multirow{2}{*}{$\begin{array}{l}\text { S. } \\
\text { No. }\end{array}$} & \multirow{2}{*}{$\begin{array}{l}\text { District/ } \\
\text { Year }\end{array}$} & \multicolumn{2}{|c|}{2006} & \multicolumn{2}{|c|}{2007} & \multicolumn{2}{|c|}{2008} & \multicolumn{2}{|c|}{2009} & \multicolumn{2}{|c|}{2010} & \multicolumn{2}{|c|}{2011} & \multicolumn{2}{|c|}{2012} & \multicolumn{2}{|c|}{2013} & \multicolumn{2}{|c|}{2014} & \multicolumn{2}{|c|}{2015} & \multicolumn{2}{|c|}{2016} & \multicolumn{2}{|c|}{2017} & \multicolumn{2}{|c|}{2018} & \multicolumn{2}{|c|}{2019} \\
\hline & & ث & 草 & U & $\stackrel{*}{*}$ & $\stackrel{*}{\cup}$ & $\stackrel{*}{*}$ & 艺 & $\stackrel{*}{*}$ & $\stackrel{*}{u}$ & $\stackrel{*}{*}$ & ש & $\stackrel{*}{*}$ & Uै & $\stackrel{*}{*}$ & 芼 & $\stackrel{*}{*}$ & ש & $\stackrel{*}{*}$ & ש & $\stackrel{*}{*}$ & $\stackrel{*}{U}$ & $\stackrel{*}{*}$ & 艺 & $\stackrel{*}{*}$ & ث & $\stackrel{*}{*}$ & $\stackrel{*}{U}$ & $\stackrel{*}{*}$ \\
\hline 1. & Deoria & 3 & 0 & 2 & 0 & 3 & 0 & 1 & 0 & 1 & 0 & 1 & 0 & 2 & 0 & 2 & 0 & & & 5 & 0 & 8 & 0 & 74 & 0 & 28 & 0 & 29 & 0 \\
\hline 2. & Kushinagar & 48 & 0 & 18 & 0 & 9 & 0 & 5 & 0 & 6 & 0 & 2 & 0 & & & 7 & 1 & 11 & 0 & 108 & 0 & 79 & 0 & 89 & 0 & 46 & 0 & 30 & 0 \\
\hline 3. & Ballia & 16 & 0 & 15 & 0 & 16 & 0 & 6 & 1 & 3 & 0 & 2 & 1 & & & & & & & 15 & 0 & 13 & 0 & 26 & 0 & 38 & 0 & 34 & 0 \\
\hline 4. & Mau & & & & & & & & & & & & & & & & & & & & & & & 1 & 0 & & & & \\
\hline 5. & Varanasi & 3 & 0 & 22 & 1 & 3 & 0 & 5 & 0 & 4 & 0 & 2 & 0 & & & & & & & & & & & & & 1 & 0 & 3 & 0 \\
\hline 6. & Ghazipur & & & 9 & 0 & & & & & & & 3 & 0 & 3 & 0 & 2 & 0 & & & 2 & 0 & 2 & 0 & 3 & 0 & 5 & 0 & 2 & 0 \\
\hline 7. & Jaunpur & 3 & 0 & & & 3 & 0 & & & & & & & & & & & & & & & 1 & 0 & & & 1 & 0 & & \\
\hline 8. & Chandauli & & & & & & & & & & & & & & & & & & & & & & & & & & & & \\
\hline 9. & Bhadohi $^{\#}$ & 7 & 0 & & & 7 & 0 & & & & & 1 & 0 & & & & & & & & & & & & & & & 1 & 0 \\
\hline 10. & Mirzapur & & & & & & & & & & & & & & & & & & & & & & & & & & & & \\
\hline 11. & Gorakhpur & & & & & & & & & & & & & & & & & & & & & 1 & 0 & & & & & & \\
\hline 12. & Ayodhya $^{* * *}$ & & & & & & & & & & & & & & & & & & & & & & & & & & & & \\
\hline 13. & Sultanpur & & & & & & & & & & & & & & & & & & & 1 & 0 & 3 & 0 & & & 2 & 0 & & \\
\hline 14. & Gonda & 3 & 0 & 3 & 0 & 3 & 0 & & & & & & & & & & & & & & & & & & & & & & \\
\hline 15. & Bahraich & & & & & & & & & & & & & & & & & & & & & & & & & & & 1 & 0 \\
\hline \multirow[t]{3}{*}{16.} & Rampur & & & & & & & & & & & & & & & & & & & & & & & & & & & & \\
\hline & Total & 83 & 0 & 69 & 1 & 44 & 0 & 17 & 1 & 14 & 0 & 11 & 1 & 5 & 0 & 11 & 1 & 11 & 0 & 131 & 0 & 107 & 0 & 193 & 0 & 121 & 0 & 100 & 0 \\
\hline & $\begin{array}{l}\text { No. of Districts } \\
\text { reported KA case }\end{array}$ & \multicolumn{2}{|c|}{7} & \multicolumn{2}{|c|}{6} & \multicolumn{2}{|c|}{7} & \multicolumn{2}{|c|}{4} & \multicolumn{2}{|c|}{4} & \multicolumn{2}{|c|}{6} & \multicolumn{2}{|c|}{2} & 3 & & 1 & & 5 & & 7 & & 5 & & . & & & \\
\hline
\end{tabular}


Table 2.District wise \& Blockwise prevalence of Kala-azar Cases in Uttar Pradesh

\begin{tabular}{|c|c|c|c|c|c|c|c|c|c|c|c|c|c|c|}
\hline \multirow[b]{2}{*}{$\begin{array}{l}\text { S. } \\
\text { No. }\end{array}$} & \multirow[b]{2}{*}{ District } & \multirow[b]{2}{*}{ Year } & \multirow{2}{*}{$\begin{array}{l}\text { Popu- } \\
\text { lation }\end{array}$} & \multicolumn{2}{|l|}{ Block/CHC } & \multicolumn{2}{|c|}{ Town ward/ village } & \multicolumn{3}{|c|}{ No. of kala-azar cases } & \multicolumn{3}{|c|}{$\begin{array}{c}\text { Prevalence index of ka/10000 } \\
\text { population at }\end{array}$} & \multirow{2}{*}{$\begin{array}{c}\text { No. of } \\
\text { pkdl } \\
\text { cases } \\
\text { repo- } \\
\text { rted }\end{array}$} \\
\hline & & & & Name & $\begin{array}{l}\text { Popul } \\
\text {-ation }\end{array}$ & Name & $\begin{array}{l}\text { Popul- } \\
\text { ation }\end{array}$ & $\begin{array}{l}\text { Dis- } \\
\text { trict }\end{array}$ & $\begin{array}{l}\text { Block/ } \\
\text { CHC }\end{array}$ & $\begin{array}{c}\text { Town } \\
\text { ward/ } \\
\text { village }\end{array}$ & $\begin{array}{l}\text { Dis- } \\
\text { trict }\end{array}$ & $\begin{array}{l}\text { Block/ } \\
\text { CHC }\end{array}$ & $\begin{array}{l}\text { Town ward } \\
\text { /village }\end{array}$ & \\
\hline \multirow[t]{12}{*}{1.} & \multirow{12}{*}{ Ballia } & \multirow{3}{*}{$\begin{array}{c}2016 \\
(06 / 17)\end{array}$} & \multirow{3}{*}{3308751} & Bariya/Kotwa & 182263 & & & \multirow{3}{*}{13} & 5 & & \multirow{3}{*}{0.04} & 0.27 & 0.00 & 0 \\
\hline & & & & Reoti & 194520 & Reoti & 40000 & & 3 & & & 0.15 & 0.75 & 0 \\
\hline & & & & - & - & - & - & & - & - & & - & - & - \\
\hline & & \multirow{3}{*}{$\begin{array}{c}2017 \\
(08 / 17)\end{array}$} & \multirow{3}{*}{3592107} & Bariya/ Kotwa & 182263 & Sukhpura & 1165 & \multirow{3}{*}{26} & 4 & 2 & \multirow{3}{*}{0.07} & 0.22 & 17.17 & 1 \\
\hline & & & & Reoti & 194520 & Reoti & 40000 & & 9 & 8 & & 0.46 & 2.00 & 1 \\
\hline & & & & M.Chhapra & 152943 & Thekaha & 1502 & & 2 & 1 & & 0.13 & 6.66 & 1 \\
\hline & & \multirow{3}{*}{$\begin{array}{c}2018 \\
(08 / 17)\end{array}$} & \multirow{3}{*}{3654447} & Bariya/Kotwa & 182263 & Bind kaTola & 1485 & \multirow{3}{*}{38} & 10 & 0 & \multirow{3}{*}{0.10} & 0.55 & 0.00 & 4 \\
\hline & & & & M.Chhapra & 152943 & Thekaha & 1502 & & 7 & 5 & & 0.46 & 33.29 & 0 \\
\hline & & & & Reoti & 194520 & Reoti & 40000 & & 6 & 5 & & 0.31 & 1.25 & 10 \\
\hline & & \multirow{3}{*}{$\begin{array}{c}2019 \\
(11 / 17)\end{array}$} & \multirow{3}{*}{3717869} & Bariya/Kotwa & 182263 & Bind kaTola & 1485 & \multirow{3}{*}{34} & 11 & 6 & \multirow{3}{*}{0.09} & 0.60 & 40.40 & 2 \\
\hline & & & & Murlichapra & 152943 & Thekaha & 1502 & & 7 & 7 & & 0.46 & 46.60 & 1 \\
\hline & & & & Reoti & 194520 & Reoti & 40000 & & 5 & 5 & & 0.26 & 1.25 & 3 \\
\hline \multirow[t]{8}{*}{2} & \multirow{8}{*}{$\begin{array}{l}\text { Kushi } \\
\text { Nagar }\end{array}$} & \multirow{4}{*}{$\begin{array}{c}2016 \\
(07 / 15)\end{array}$} & \multirow{4}{*}{3999025} & Taryasujan & 303236 & Basdila Bujurg & 3826 & & 29 & 0 & & 0.96 & 0.00 & 0 \\
\hline & & & & Tamkuhi & 294515 & & & 79 & 22 & - & בית & 0.75 & - & 0 \\
\hline & & & & Padrauna/ Kubernath & 387092 & Nahar Chhapra & 7140 & 19 & 11 & 3 & 0.20 & 0.28 & 4.20 & 0 \\
\hline & & & & Dudhi & 289416 & Guruliya & & & 12 & 2 & & 0.41 & $>1$ & 0 \\
\hline & & & & Taryasujan & 303236 & Basdila Bujurg & 3900 & & 27 & 0 & & 0.89 & 0.00 & 0 \\
\hline & & 2017 & & Tamkuhi & 294515 & & & & 41 & - & & 1.39 & - & 0 \\
\hline & & $(08 / 15)$ & 4092081 & Padrauna/ Kubernath & 387092 & N.Chhapra & 7280 & 89 & 14 & 7 & 0.22 & 0.36 & 9.62 & 4 \\
\hline & & & & Dudhi & 289416 & Guruliya & & & 3 & 0 & & 0.10 & 0.00 & 0 \\
\hline
\end{tabular}




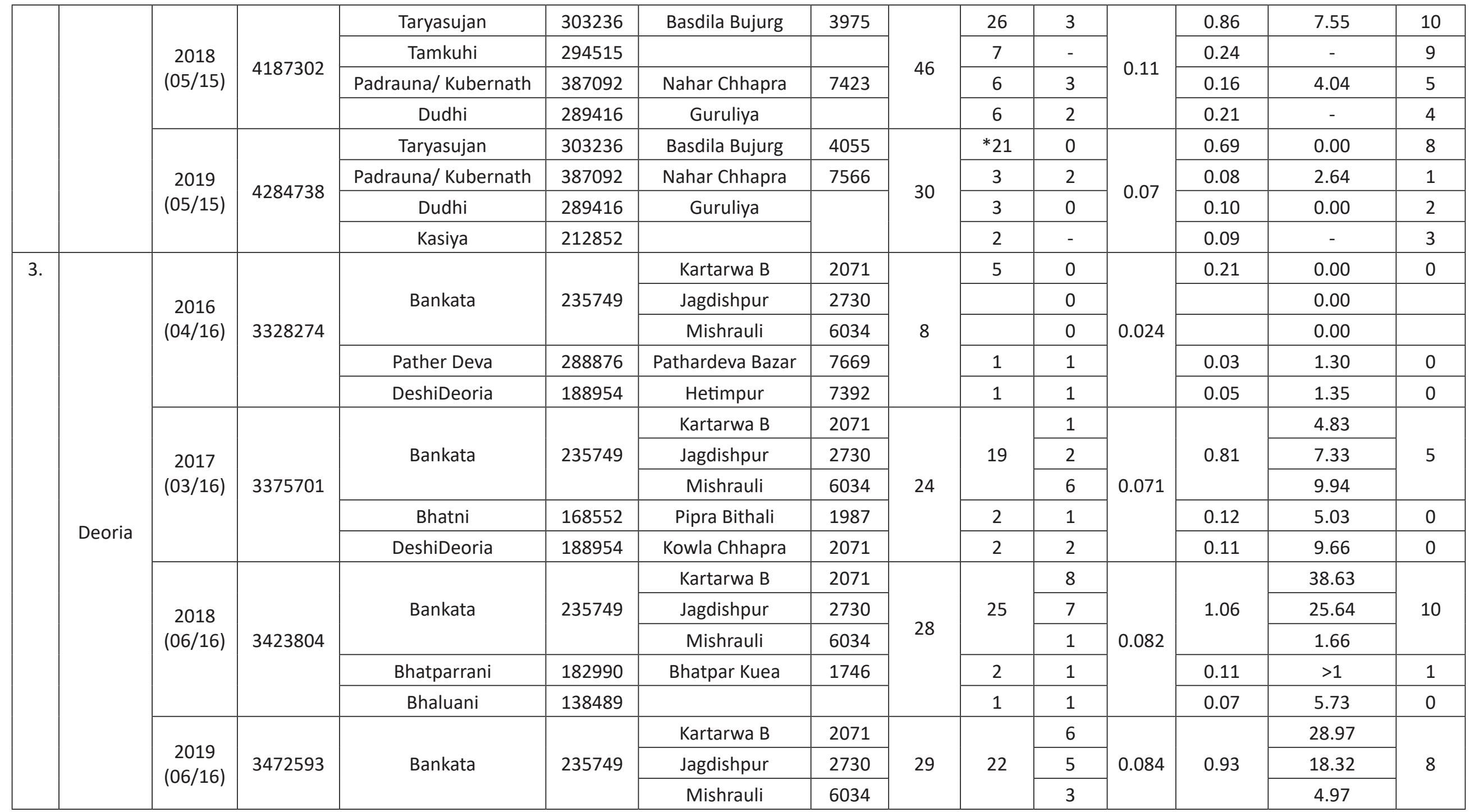


Chandra $R$ et al.

J. Commun. Dis. 2021; 53(1)

\begin{tabular}{|c|c|c|c|c|c|c|c|c|c|c|c|c|c|c|}
\hline & & & & Bhatparrani & 182990 & Aktahi Bazar & 2856 & & 2 & 1 & & 0.11 & 3.50 & 0 \\
\hline & & & & Pather Deva & 288876 & Laxmipur & 1398 & & 1 & 1 & & 0.03 & 7.15 & 2 \\
\hline \multirow[t]{4}{*}{4} & \multirow{4}{*}{ Varanasi } & $\begin{array}{c}2016 \\
(00 / 10)\end{array}$ & 4003061 & \multicolumn{11}{|l|}{ NIL } \\
\hline & & $\begin{array}{c}2017 \\
(00 / 10)\end{array}$ & 4071700 & \multicolumn{11}{|l|}{ NIL } \\
\hline & & $\begin{array}{c}2018 \\
(01 / 10)\end{array}$ & 4141515 & Sewapuri & 240184 & Arjunpur & 2140 & 1 & 1 & 1 & 0.002 & 0.042 & 4.67 & 0 \\
\hline & & $\begin{array}{c}2019 \\
(01 / 10)\end{array}$ & 4212527 & K.V.Pith & 238793 & Harpalpur & 7710 & 3 & 2 & 2 & 0.007 & 0.08 & 2.59 & 2 \\
\hline \multirow[t]{12}{*}{5.} & \multirow{12}{*}{$\begin{array}{l}\text { Ghaz- } \\
\text { ipur }\end{array}$} & \multirow{2}{*}{$\begin{array}{c}2016 \\
(02 / 16)\end{array}$} & \multirow{2}{*}{3981078} & Moham-madabad & 221471 & Faizullapur & 1500 & 2 & 1 & 1 & 0.005 & 0.045 & 6.67 & 0 \\
\hline & & & & Manihari & 217280 & & & & 1 & 1 & & 0.046 & $>1$ & 0 \\
\hline & & \multirow{2}{*}{$\begin{array}{c}2017 \\
(02 / 16)\end{array}$} & \multirow{2}{*}{4057445} & Moham-madabad & 221471 & Faizullapur & 1500 & 3 & 2 & 2 & 0.007 & 0.09 & 13.33 & 0 \\
\hline & & & & Gournour & 188989 & & & & 1 & 1 & & 0.05 & $>1$ & 0 \\
\hline & & \multirow{4}{*}{$\begin{array}{c}2018 \\
(03 / 16)\end{array}$} & \multirow{4}{*}{4135277} & Moham-madabad & 221471 & Faizullapur & 1500 & 5 & 3 & 1 & 0.012 & 0.13 & 6.67 & 1 \\
\hline & & & & & & Raghuvarganj & 2750 & & & 2 & & & 7.27 & \\
\hline & & & & Gournour & 188989 & & & & 1 & 1 & & 0.05 & $>1$ & 0 \\
\hline & & & & Barachawar & 204173 & & & & 1 & 1 & & 0.05 & $>1$ & 0 \\
\hline & & \multirow{4}{*}{$\begin{array}{c}2019 \\
(02 / 16)\end{array}$} & \multirow{4}{*}{4214602} & Moham-madabad & 221471 & Faizullapur & 1500 & $\begin{array}{c}2+5 \\
(\mathrm{PKDL})\end{array}$ & 3 & 1 & 0.016 & 0.135 & 6.67 & 2 \\
\hline & & & & & & Raghuvarganj & 2750 & & & 2 & & & 7.27 & \\
\hline & & & & Gournour & 188989 & & & & 1 & 1 & & 0.05 & $>1$ & 1 \\
\hline & & & & Mardah & 186435 & & & & 2 & 1 & & 0.11 & $>1$ & 2 \\
\hline
\end{tabular}

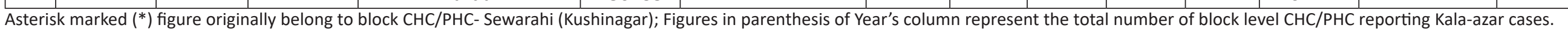


The state is much serious about the elimination of the disease to achieve the goal by 2020 . The KA cases are being searched regularly in special drives in endemic districts, along with districts reporting sporadic districts. The KA cases are being treated completely with Am Bisome (Liposomal Amphotericin B) injection in single dose according to body weight $(10 \mathrm{mg} / \mathrm{kg}$ ) but it is not sure, whether whole district or the KA affected area has been searched thoroughly or not, because many new areas of the affected districts have been reported in consecutive drives leaving behind the already affected area (Table2). Moreover, Post Kala-Azar Dermal Leishmaniasisis (PKDL) cases are also being reported in adequate number in these search drives during last few years (Table $2 \& 3$ ), which are being treated too but the matter of much concern is, that these PKDL cases may act as reservoir of infection of the disease, if left unsearched and untreated. Such areas may be supplemented with the KA cases, which were left for survey having no report of KA cases, as is being practiced not to conduct survey in the complete district, together with recent infection having long intrinsic incubation period, even for more than a year ${ }^{51}$ and those reported in the hospitals, when the patients approached for their treatment. The remaining districts with sporadic occurrence of KA cases, the active case search drives are restricted to only limited areas, which cannot be assumed to be a right step, when the disease is subjected for elimination and vector has been reported from many parts of India. ${ }^{11-50}$

It is pertinent to mention here that the disease elimination goal has been targeted as < one KA case/ 10000 population of health unit at block level. If, we consider the whole block population as denominator to total KA cases as numerator to find out it at 10000 fraction, then the resultant outcome is $<1 / 10000$ during last four years except block/CHC/ PHC-Tamkuhi of District Kushinagar and block/CHC/PHCBankata of District Deoria, which reflected KA prevalence $1.39 / 10000$ \& 1.06/10000, respectively. But at district level, the KA prevalence becomes much below $<1 / 10000$. Thus, there is no need to conduct any intervention activity towards the elimination of the disease on considering the block as a unit but the same are being carried out with active involvement/ participation of the Non-Government Organizations NGOs).The basic health unit for implementing various health programmes at peripheral level, Health Sub-Centre (HSC), with a population of 5000 in plain \& 3000 in hilly or hard to reach areas (erstwhile the health worker area with population of 10000 under National Malaria Eradication Programme), HSC has been subjected as basic health unit for identifying the high risk malaria area and other purposes. If existing health unit (HSC) is considered for elimination of the disease, with HSC not more than two in number, will make a population of 10000 , the fraction of the population at which elimination of the disease may be ensured but at present majority of the HSCs have population $>10000$ and single HSC may be sufficient to be considered for elimination. If, health subcenter (HSC), is considered as basis unit with population of 10000 , many more health sub-centers, may reflect $K A$ cases $>1$ per 10000 population or even combining two health sub centers' 5000 population, at which HSCs were established/ created as is evident from Table 2, wherein the village/ town or health sub-centers of five districts, Deoria, Kushinagar, Ballia, Ghazipur and Varanasi reporting regularly KA cases, reflect $>1 \mathrm{KA}$ case/ 10000 population (up to $46.60 \mathrm{KA}$ cases/ 10000 population) and need special attention of the state/ district health programme officers for undertaking intensified intervention measures for: (a) active cases search drive for at least three years sweeping the whole population of the district and (b) complete treatment of the KA cases (both VL \& PKDL).

\section{Indoor Residual Spray (IRS)}

IRS is recommended in the programme against vector Phlebotomusargentipes with alphacypermethr in 5\% wp since 2017, prior to this DDT 50\% wdp was sprayed in the programme. The IRS is effected inside the human dwellings \& cattle sheds in accordance with the prescribed time schedule and good quality, leaving no sign of partial \& patchy coverage of the spray. The dose of the insecticide is to be monitored along with the discharge rate and under dose of the insecticide should not be practiced.

Table 3.Post Kala-azar dermal Leishmaniasisis (PKDL) cases in Uttar Pradesh

\begin{tabular}{|c|c|c|c|c|c|c|c|c|}
\hline \multirow{2}{*}{$\begin{array}{c}\text { S. } \\
\text { No. }\end{array}$} & \multirow{2}{*}{ Year } & \multicolumn{2}{|c|}{ No. of PKDL } & \multicolumn{2}{c|}{ No. of reporting PKDL } & Population of PKDL & \multirow{2}{*}{$\begin{array}{c}\text { PKDL/10000 } \\
\text { population }\end{array}$} \\
\cline { 3 - 9 } & & Cases & Deaths & Districts & Cases PHC & Districts & PHCs & 0.0929 \\
\hline 1. & 2017 & 12 & 0 & 3 & 5 & 11265553 & 1291718 & 0.1471 \\
\hline 2. & 2018 & 70 & 0 & 7 & 23 & 25535801 & 4691995 & 0.14719 \\
\hline 3. & 2019 & 52 & 1 & 6 & 24 & 24285819 & 5211171 & 0.0979 \\
\hline
\end{tabular}


Table 4.Indoor Residual Spray (IRS) activities conductedin Uttar Pradesh during last three years

\begin{tabular}{|c|c|c|c|c|c|c|c|c|c|c|c|c|c|c|c|c|c|c|c|}
\hline \multirow{3}{*}{$\begin{array}{l}\text { S. } \\
\text { No. }\end{array}$} & \multirow{3}{*}{ District } & \multicolumn{6}{|c|}{ IRS Done } & \multicolumn{6}{|c|}{ I $^{\text {st }}$ Round IRS Coverage (\%) } & \multicolumn{6}{|c|}{ III Round IRS Coverage (\%) } \\
\hline & & \multicolumn{2}{|c|}{2017} & \multicolumn{2}{|c|}{2018} & \multirow[b]{2}{*}{$\begin{array}{c}\text { Ist }^{\text {st }} \\
\text { Round }\end{array}$} & \multirow[b]{2}{*}{$\begin{array}{c}\text { II nd }^{\text {nound }} \\
\text { Round }\end{array}$} & \multicolumn{3}{|c|}{ Houses } & \multicolumn{3}{|c|}{ Rooms } & \multicolumn{3}{|c|}{ Houses } & \multicolumn{3}{|c|}{ Rooms } \\
\hline & & $\begin{array}{c}\text { It }^{\text {st }} \\
\text { Round }\end{array}$ & $\begin{array}{c}\text { II nd }^{\text {nound }} \\
\text { Round }\end{array}$ & $\begin{array}{c}\text { It }^{\text {st }} \\
\text { Round }\end{array}$ & $\begin{array}{c}I^{\text {nd }} \\
\text { Round }\end{array}$ & & & 2017 & 2018 & 2019 & 2017 & 2018 & 2019 & 2017 & 2018 & 2019 & 2017 & 2018 & 2019 \\
\hline 1. & Deoria & $\begin{array}{l}\text { June- } \\
\text { Jul. }\end{array}$ & $\begin{array}{l}\text { Oct.- } \\
\text { Dec. }\end{array}$ & ND & ND & $\begin{array}{c}\text { March- } \\
\text { May }\end{array}$ & $\begin{array}{l}\text { Sept.- } \\
\text { Nov. }\end{array}$ & 34.84 & 32.67 & 67.57 & 28.76 & ND & 62.15 & 90.61 & ND & 96.77 & 92.86 & ND & 96.55 \\
\hline 2. & $\begin{array}{l}\text { Kushi- } \\
\text { nagar }\end{array}$ & $\begin{array}{l}\text { June- } \\
\text { Sept. }\end{array}$ & $\begin{array}{l}\text { Sept.- } \\
\text { Nov. }\end{array}$ & $\begin{array}{l}\text { March- } \\
\text { May }\end{array}$ & ND & $\begin{array}{c}\text { March- } \\
\text { July }\end{array}$ & $\begin{array}{l}\text { Sept.- } \\
\text { Nov. }\end{array}$ & 97.00 & 70.00 & 94.23 & 98.00 & 75.00 & 99.30 & 89.36 & ND & 66.75 & 84.06 & ND & 72.13 \\
\hline 3. & Ballia & $\begin{array}{l}\text { Jul.- } \\
\text { Sept. }\end{array}$ & ND & ND & ND & $\begin{array}{c}\text { March- } \\
\text { July }\end{array}$ & $\begin{array}{l}\text { Oct.- } \\
\text { Jan. }^{20}\end{array}$ & 99.00 & 100.00 & 149.24 & 99.00 & & 99.38 & ND & ND & 183.34 & ND & ND & 140.88 \\
\hline 4. & $\begin{array}{l}\text { Ghazi- } \\
\text { pur }\end{array}$ & $\begin{array}{l}\text { June- } \\
\text { Jul. }\end{array}$ & $\begin{array}{l}\text { Oct.- } \\
\text { Nov. }\end{array}$ & $\begin{array}{l}\text { April- } \\
\text { May }\end{array}$ & ND & \begin{tabular}{|c|} 
March- \\
April
\end{tabular} & $\begin{array}{c}\text { Octo- } \\
\text { ber }\end{array}$ & 97.87 & 49.67 & 116.53 & 88.52 & 42.33 & 102.57 & 97.47 & ND & 51.09 & 81.64 & ND & 45.36 \\
\hline 5. & $\begin{array}{c}\text { Gorakh- } \\
\text { pur }\end{array}$ & July & $\begin{array}{l}\text { Nov- } \\
\text { ember }\end{array}$ & ND & ND & ND & $\begin{array}{l}\text { Nov- } \\
\text { ember }\end{array}$ & 100.00 & 100.00 & 0.00 & 100.00 & & 0.00 & 100.00 & ND & 98.49 & 100.00 & ND & 98.54 \\
\hline 6. & Jaunpur & $\begin{array}{l}\text { Mar- } \\
\text { Jun. }\end{array}$ & $\begin{array}{c}\text { Sep- } \\
\text { tember }\end{array}$ & April & ND & ND & $\begin{array}{l}\text { Nov- } \\
\text { ember }\end{array}$ & 100.00 & 97.63 & 0.00 & 100.00 & 60.68 & 0.00 & 95.86 & ND & 100.00 & 77.88 & ND & 92.72 \\
\hline 7. & Varanasi & NA & NA & ND & ND & $\begin{array}{l}\text { Jul.- } \\
\text { Aug. }\end{array}$ & $\begin{array}{l}\text { Sept.- } \\
\text { Oct. }\end{array}$ & NA & NA & 70.90 & NA & & 46.51 & NA & ND & 100.00 & NA & ND & 88.51 \\
\hline 8. & $\begin{array}{l}\text { Sultan- } \\
\text { pur }\end{array}$ & March & $\begin{array}{l}\text { Aug.- } \\
\text { Sept. }\end{array}$ & ND & ND & ND & $\begin{array}{l}\text { Dece- } \\
\text { mber }\end{array}$ & 65.06 & 100.00 & 0.00 & 55.48 & & 0.00 & 85.78 & ND & 99.34 & 97.63 & ND & 90.22 \\
\hline 9. & Bhadohi & NA & NA & ND & ND & ND & $\begin{array}{l}\text { Nov- } \\
\text { ember }\end{array}$ & NA & NA & 0.00 & NA & NA & 0.00 & NA & ND & 92.92 & NA & ND & 83.21 \\
\hline \multirow[t]{2}{*}{10.} & Mau & NA & NA & ND & ND & ND & $\begin{array}{c}\text { Octo- } \\
\text { ber }\end{array}$ & NA & NA & 0.00 & NA & NA & 0.00 & NA & ND & 93.57 & NA & ND & 91.95 \\
\hline & Total & $\begin{array}{l}\text { Mar.- } \\
\text { Sept. }\end{array}$ & $\begin{array}{l}\text { Aug.- } \\
\text { Dec. }\end{array}$ & $\begin{array}{l}\text { Mar.- } \\
\text { May }\end{array}$ & ND & $\begin{array}{l}\text { Mar.- } \\
\text { Aug. }\end{array}$ & $\begin{array}{l}\text { Sept.- } \\
\text { Jan. }^{20}\end{array}$ & & & 99.81 & & & 86.71 & & & 100.40 & & ND & 95.07 \\
\hline
\end{tabular}

$\mathrm{ND}=$ Not done and NA=Not applicable.

ISSN: 0019-5138

DOI: https://doi.org/10.24321/0019.5138.202108 
The monitoring of the IRS activities revealed (Table 4) that time schedule was not followed in the districts as the $1^{\text {st }}$ round of IRS was performed from March to September during 2017, 2018 \& 2019 instead of scheduled time of February/ March. The IInd round of IRS was performed from September to January of the ensuing year during these years instead of scheduled time of June/ July but the IInd round of IRS was not performed in 2018. The reported room coverage varied from $28.76 \%$ to $183.34 \%$, reflecting extreme variation. The low coverage of rooms below $90 \%$ may not have the desired impact against the vector and similarly the over coverage reported by district Ballia may not provide the desired dose of the insecticide as, house \& room coverage reported by District Ballia in 2019 remained $149.24 \% \& 99.38 \%$ in $1^{\text {st }}$ round and $183.34 \%$ \& $140.88 \%$ in $2^{\text {nd }}$ round, whereas house and room coverage reported by District Ghazipur in 2019 remained $116.53 \% \& 102.57 \%$ in $1^{\text {st }}$ round. It is worth mentioning when the exact quantity of the insecticide has been provided as per proposed IRS plan, for covering the fixed population, the over coverage will ultimately give under dose of the insecticide to kill the vector, making it a futile exercise of IRS and undue expenditure incurred on it is nothing but merely wastage. Moreover, partial exposure to the insecticide sprayed and the sub-lethal doses of the insecticide applied against the vector, may force to develop resistance against the insecticide in due course as has been reported in Phlebotamus argentipes and Phlebotomus papatassi in Bihar Gujarat and Uttar Pradesh. ${ }^{52-54}$ Hence, to achieve the goal of elimination of the disease, the state health authorities need to ensure: (a) complete coverage of population with IRS following the time line and quality of IRS as per NVBDCP guidelines, and (b) The intervention activities need to be synchronized with neighboring states or country subjecting their areas for KA elimination.

\section{Conclusion}

On the basis of foregoing observations and discussion, it can be inferred that the programme of elimination of the KA disease is being implemented in the state but the guidelines framed and issued by Government of India (NVBDCP) are not being followed strictly. The target of elimination of KA disease could have been achieved, if the sub-district or block area is considered as an unit and need not to undertake further intervention measures but the target achievement is beyond reach, if village/ sub-centers are considered as basic elimination unit and the programme activities in these districts are to be implemented seriously \& monitored closely. The absence of dedication towards programme will not only deprive from achieving the goal of elimination of KA but will pose a threat of spread of KA disease together with cutaneous leishmaniasis, reported from south Indian states. The presence of vector has been reported from many parts of India and likely may increase the problem of these disease. In addition to theses, an aggressive strategy in form of Jan Andolan by Social mobilization is also required for elimination of the disease. Further in endemic districts, an enhanced Active Case Search (ACS) and quality indoor residual spray (IRS) asper the time schedule of NVBDCP apart from massive IEC activities are needed to eliminate this disease from India.

\section{Acknowledgement}

The authors are thankful to State Vector Borne Disease Programme Officer, Directorate General of Medical \& Health Services, Lucknow (UP) and District Malaria/ VBD Officers/ Health authorities of Kala-azar endemic districts of the State for their inputs and support rendered for completing the present task.

\section{Conflict of Interest: None}

\section{References}

1. Lal S, Saxena NBL, Dhillon GPS. Manual on Visceral Leishmaniasisis (Kala-Azar) in India. National Malaria Eradication Programme 1996; 1-182.

2. Sehgal S, Bhatia R. Plague, National Institute of Communicable Diseases. Directorate General of Health Services, Delhi. 1994; 1-64.

3. Banerjee KB. Kala-azar in India with particular reference to Bihar. Proc. on Vector Borne Diseases and Control (211 November, 1987), NICD, Delhi, DGHS, Government of India. 1989; 20-22.

4. National Plan for Regional Kala-azar Elimination Programme, India.Directorate of National Vector Borne Disease Control Programme, DGHS, Ministry of Health \& Family Welfare, 2005; 39.

5. National Consultative workshop on National Vector Borne Disease Control Programme, Government of India, DGHS, Ministry of Health \&Family Welfare, 2005; 24.

6. Jung RK. Epidemiological situation and control of Malaria and Kala-azar in intercountry bordering areas of Bangladesh, Bhutan, India and Nepal. WHO/SEARO, 1998; 1-109.

7. Operational guidelines on Kala-azar (Visceral Leishmaniasisis) elimination in India. World Health Organization, Geneva. 2015.

8. Operational Guidelines on Kala-azar (Visceral Leishmaniasisis) Elimination in India.National Malaria Eradication Programme, 2015; 1-99.

9. Guidelines on Vector Control in Kala-azar Elimination. National Malaria Eradication Programme. 2015; 21.

10. Accelerated Plan for Kala-azar Elimination. National Malaria Eradication Programme. 2017; 80.

11. Adler $S$, Theodor $O$. Transmission of disease agent by Phlebotamine sandflies. Ann Rev Ent 1957; 2: 203-226.

12. Bhattacharya PK. Distribution of Kala-azar in India. Trans 
Roy Soc Trop Med \& Hyg 1981; 75: 333-334.

13. Smith ROA, Lal C, Mukerjee $S$ et al. Bionomics of Phlebotamusargentipes. Part I: Duration of life in nature. Indian J Med Res 1936; 24: 295-300.

14. Chowdhury AB. Recent resurgence of Kala-azar in West Bengal. Proc. of Indo-UK Workshop on Leishmaniasisis held at Patna. Indian Council of Medical Research, New Delhi. 1983; 10-19.

15. Dhanda V, Gill GS. Double blood meals by Phlebotamusargentipes and Phlebotamuspapatasi in two villages of Maharashtra. Indian J Med Res 1982; 76: 840-2.

16. Dhanda V, Modi GBC. Studies on the sandflies collected indoors from Aurangabad district, Maharashtra State (Diptera: Psychodidae). Indian J Med Res 1971; 59: 1565-71.

17. Dhanda V, Shetty PS, Dhiman RC. Studies on Phlebotamine sandflies as vectors of Kala-azar in Bihar Proc. of Indo-UK Workshop on Leishmaniasisis held at Patna. Indian Council of Medical Research, New Delhi.1983; 128-137.

18. Hati AK. Current status of Leishmaniasisis vector biology.Proc. of Indo-UK Workshop on Leishmaniasisis held at Patna. Indian Council of Medical Research, New Delhi. 1983; 84-91.

19. Killick-Kendrick R.Investigation of Phlebotaminae sandflies-vectors of Leishmaniasisis.Proc. of Indo-UK Workshop on Leishmaniasisis held at Patna. Indian Council of Medical Research, New Delhi. 1983; 72-83.

20. Pandya AP. Laboratory breeding of the sandflies, Phlebotamus argentipesAnnandale \& Brunetti and Phlebotamuspapatasi. Indian J Med Res 1980; 72: 64-5.

21. Kumar K, Rahman SJ, Singh K et al. Biology of Phlebotamusand Sergentomyiagroup of sand flies under laboratory conditions. J Comm Dis 1986; 18: 45-50.

22. Kalra NL, Kumar K, Rahman SJ. Sandflies of bird tunnel in Eastern mounds from district Rohtak (Haryana). J Comm Dis 1986; 18: 136-138.

23. Kumar K, Rahman SJ, Rajagopal R. Phlebotamid sandflies (Diptera: Phlebotaminae) in Delhi and Environs. J Comm Dis 1988; 20: 44-51.

24. Srinivasan R. Studies on the biology, ecology and population dynamics of Phlebotamuspapatasi (Diptera: Phlebotamidae) in Pondicherry. Ph.D. thesis submitted to Pondicherry University, 1990; 164.

25. Srinivasan R, Viswam K, Panicker, KN. An improvised method of laboratory colonization of Phlebotamuspapatasi, the vector of cutaneous Leishmaniasisis. J Expt Biol 1992; 30: 925-927.

26. Kamal S, Srinivasan R. Investigations on the Biology of Phlebotamusargentipes, Annandale \& Brunetti, 1908 (Diptera: Phlebotamidae), the vector of Indian Kala- azar. J Comm Dis 1993; 25(3): 96-100.

27. Kaul SM. Phlebotamine sandflies (Diptera: Psychodidae) from Western Ghats in Kerala and Tamil Nadu States, India, Part I. Description of four new species. J Comm Dis 1993; 25(2): 75-82.

28. Kaul SM, Das RK, Shiv R et al. Entomological monitoring of Kala-azar control in Bihar State, India: Observations in Vaishali and Patna districts. J Comm Dis 1993; 25(3): 101-106.

29. Kaul SM. Phlebotamine sandflies (Diptera: Psychodidae) from Western Ghats in Kerala and Tamil Nadu States, India, Part II. Taxonomic and biological notes on the species recorded. J Comm Dis 1993; 25(3): 116-125.

30. Kaul SM, Prabha PGS. Phlebotamine sandflies (Diptera: Psychodidae) from Western Ghats in Kerala and Tamil Nadu States, India, Part III. Description of Sergentomyiaarboris Sinton and new species related to it. J Comm Dis 1993; 25(4): 199-206.

31. Swaminath CS, Shortt HE, Andersan LAP. Transmission of Indian Kala-azar to man by the bites of P. argentipes (Ann. \&Brun.). Indian J Med Res 1942; 30: 473-477.

32. Sinton JA. Records of geographical distribution and seasonal prevalence of known Indian and Cingrales of the genus Phlebotamus. Indian J Med Res 1924; 11: 55-74.

33. Rathnaswamy GK, Ramkrishnan NR. Report on the sand fly survey of Madras city. Indian J Ent 1954; 16: 29-36.

34. Shetty PS, Kulkarni SM. Records of Phlebotamussandflies ofBastar district, Madhya Pradesh (India). Indian J Parasit 1983; 7: 87-88.

35. Rahman SJ, Menon PKM, Rajagopal R et al. Behaviour of Phlebotamusargentipesin the foothills of Nilgiris, Tamilnadu. J Comm Dis 1986; 18: 35-44.

36. Modi GB, Soman, RS. A note on the Phlebotamine sandflies of Krishna and Godavari districts of Andhra Pradesh. Indian J Parasit 1978; 2: 159-161.

37. Modi GB, Shetty PS, Mahadev PVM et al. Distribution and adult habitats of Phlebotamine sandflies from Maharashtra (India). J Bombay Nat His Soc 1972; 66: 845-847.

38. Modi GB, Soman RS, Mishra AC et al. A report on the Phlebotaminesandflies (Diptera: Phlebotamine) from Bankura district (West Bengal). Indian J Med Res 1977; 66: 373-378.

39. Modi GB, Dhanda V, Goverdhan RS. Studies on Phlebotamine sandflies ofPoona (Maharashtra), India. Indian J Med Res 1978; 67: 48-56.

40. Napier LE, Smith ROA. A study of the bionomics of $P$. argentipeswith special reference to conditions in Calcutta. Indian Med Res Memoirs 1926: 4: 161-172.

41. Nasiruddin M. Phlebotamusargentipesfound in Shimoga district, Mysore state (Karnataka), India. Indian Med Gaz 1951; 36: 142-145. 
42. Pandya AP, Mandal MM, Niyogi AK. Blood meal of Phlebotamus species in Gujarat. J Comm Dis 1972; 4: 132-135.

43. Pandya AP, Mehta NR, Trivedi DH. Studies on the sandflies collected indoors in Surat city and nearby two villages of Surat, Gujarat. Phlebotamus species in Gujarat. Indian J Med Res 1972; 31: 133-136.

44. Pandya AP. Geographical distribution and density of Phlebotamine sandflies in Surat district (Gujarat). Phlebotamus species in Gujarat. Indian J Med Res 1983; 77: 817-23.

45. Raman TK. Kala-azar in Madras. Madras Medical College Magazine 1927; 4: 273-294.

46. Shanmugam CAK, Roy RG, Ganesan AV. Kala-azar in Tamil Nadu during 1945-1975. A retrospective and prospective study. Indian J Med Res 1977; 65: 786-806.

47. Saini P, Kumar NP, Ajithlal PM et al. Visceral Leishmaniasisis caused by Leishmaniadonovani Zymodeme MON-37, Western Ghats, India. Research Letter Emerging Infectious Diseases 2020; 26(8): 19561958.

48. Kesavan A, Parvathy VK, Thomas S et al. Indigenous visceral Leishmaniasisis: two cases from Kerala. Indian Pediatr 2003: 40-373.

49. Kumar NP, Srinivasan R, Anish TS et al. Cutaneous Leishmaniasisis caused by Leishmaniadonovaniin the tribal population of the Agasthyamala Biosphere Reserve forest, Western Ghats, Kerala, India. J Med Microbiol 2015; 64: 157-163.

50. Srinivasan R, Kumar NP, Jambulingam P. Detection of natural infection of Leishmania donovani(Kinetoplastida: Trypanosomatidae)in Phlebotamusargentipes(Diptera: Psychodidae)from a forest ecosystem in the Western Ghats, India, endemic for cutaneous Leishmaniasisis. Acta Trop 2016; 156: 95-99.

51. Rahman SJ, Kumar K, Sharma SK. Intrinsic incubation period of kala-azar in man through entomological and epidemiological parameters-A retrospective study. Adv Med Entomol \& Human Welfare 1998; 119-123.

52. Joshi GC, Kaul SM, Wattal BL. Susceptibility of sandflies to organochlorine insecticides in Bihar (India)-Further reports. J Comm Dis 1979; 11: 213-218.

53. Thapar BR, Joshi, RD, Rao JS et al. Susceptibility status of PhlebotamuspapatasiScopoli (Diptera: Psychodidae) to chlorinated hydrocarbons in Panchamahal District of Gujarat State (India). J Comm Dis 1993; 25(4): 212-213.

54. Joshi RD, Rai RN. Impact of DDT spraying on populations of P. argentipes and P.papatasi in Varanasi District, Uttar Pradesh. J Comm Dis 1994; 26(1): 56-58. 